\title{
Survey of Routing in Delay Tolerant Networks
}

\author{
Harshad S. Modi \\ Lecturer Computer Engineering \\ Govt. polytechnic Gandhinagar
}

\author{
Nikhil Kumar Singh \\ Lecturer Computer Engineering \\ Govt. polytechnic Gandhinagar
}

\begin{abstract}
Delay Tolerant Networks (DTNs) is a network, where synchronous connectivity among all nodes doesn't exist i.e. end to end connectivity not mandatory to be present. The major problem of DTN is how to route a packet from one terminal to another. Due mobility of nodes, unreliable and temporary connectivity the routing problem becomes more complex. It is very tedious to design the routing protocol for such kind networks. This work surveys the various routing techniques.
\end{abstract}

\section{General Terms \\ Design, Performance}

\section{Keywords}

Flooding, store-carry-forward, Delay tolerant network

\section{INTRODUCTION}

Today TCP/IP protocol suite plays an important role to develop the connection and communicating between devices worldwide. This model tries to transfer the data in minimal possible delay and high reliability. The main working principals of TCP/IP protocol suit is end to end data transfer, effective route selection, retransmission policy to correct the errors etc. Conventional routing protocols do not work in a DTN because they assume end-to-end connectivity which is not guaranteed for DTN. Basically internet works on TCP/IP protocol suit which has certain assumptions [1]:

a. During the communication for a particular session, endto-end connectivity is maintained between source and destination

b. Retransmission policy is used to fix the error which is based on time and receiver

c. All routers and end stations should support the TCP/IP protocols

d. communication performance is not a parameter for applications

e. Most of the security issues are resolved at end points

f. Packet switching is the most appropriate abstraction for interoperability and performance

g. selecting a single route between sender and receiver is sufficient to get the communication performance

\subsection{What is DTN}

Delay tolerant networks (DTNs) represent a class of wireless systems that virtually need minimum to none infrastructure. DTNs are intended to deal with scenarios involving heterogeneity of standards, intermittent connectivity between adjacent nodes, lack of contemporaneous end-to-end links and exceptionally high delays and error-rates. Also the mobile nodes available in challenged environments can be extremely limited in their resources; such as CPU processing power, memory and network capacity. DTN uses a range of complex applications that include:

1. Wireless sensor networks (WSNs) deployed in wildlife tracking or in extreme regions (e.g. volcanic and underwater areas).

2. Mobile Ad-Hoc networks connecting remote and rural communities via GPSs, cellular devices and portable storages.

3. Exotic Media Networks (EMNs) interconnecting extraterrestrial nodes such as satellites and deep space probes in Inter-Planetary Networks (IPNs).

Above field of applications operate under savior circumstances and in critical environments where ordinary network setting fails.

\subsection{Characteristics of DTN}

Delay Tolerant Networks have the following Characteristics [2]:

a. Intermittent Connectivity: Where no end-to-end connectivity is present between source and destination that is network partitioning.

b. Long or Variable Delay: During the intermittent connectivity, between the nodes have long propagation delays and variable queuing delays it is end-to-end path delays.

c. Asymmetric Data Rates: In the network user use asymmetric of bidirectional data rate.

d. High Error Rates: In a link when bit errors are generated required some correction or retransmission for the entire packet where link-error rate is generated.

Figure 1 show that the destination is unavailable, which calls for a different strategy for implementing DTN's.

\subsection{Architecture of DTN}

Delay network architecture is designed as an overlay of existing networks which works on the following concepts -

\subsubsection{Bundle layer}

DTN architecture introduce an overlay just above the transport layer is called bundle layer. Bundles are also called messages. By storing and forwarding entire bundles between the nodes, data transfer takes place. The bundle comprises with source nodes, user-data, control information, a bundle header. This layer is already easily linked with TCP/IP to provide a gateway when two nodes come to contact with each other. Flexibility is the major advantage [3]. 


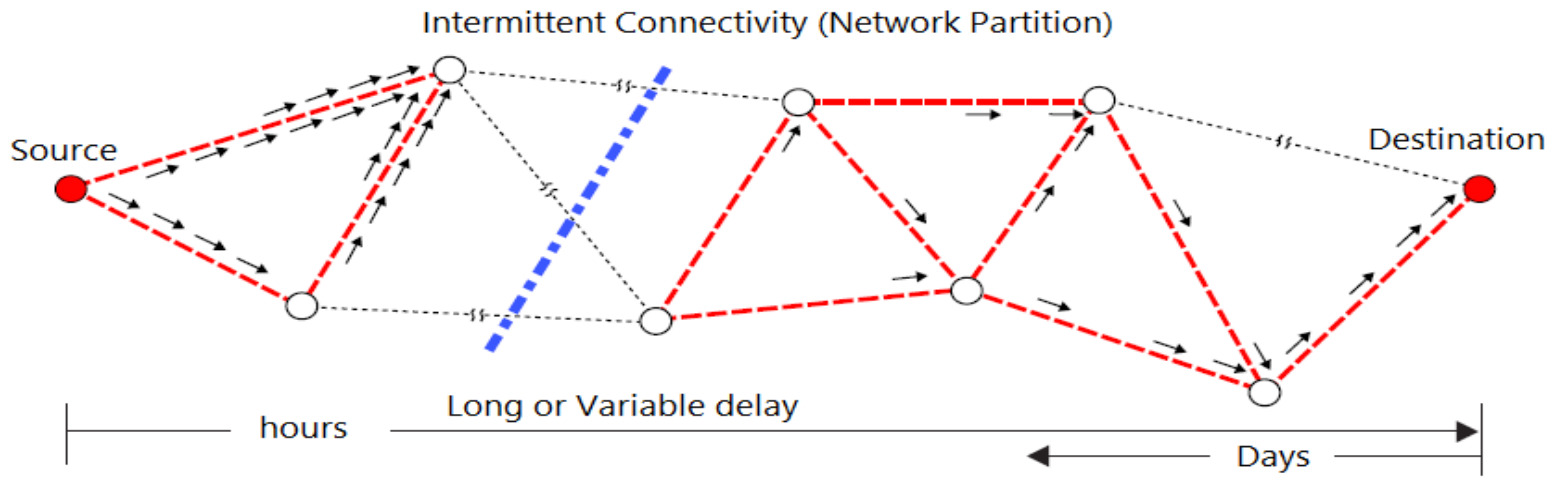

Assymetric data rates

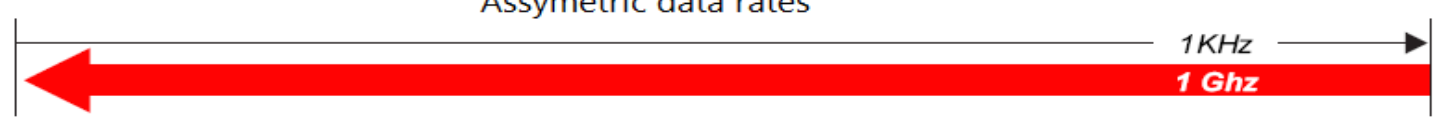

High Error Rates

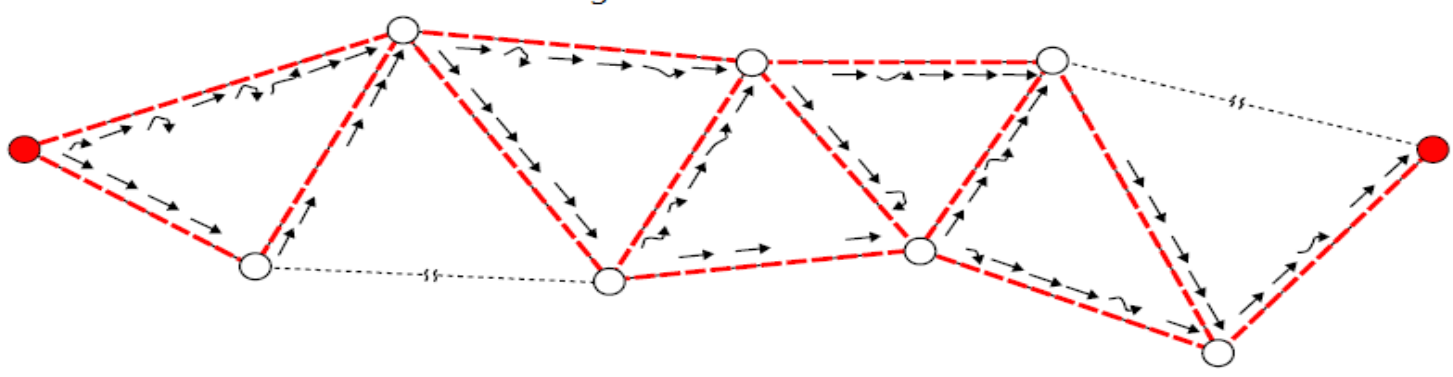

Fig 1: Intermittency and High Error Rates in the DTN network [2]

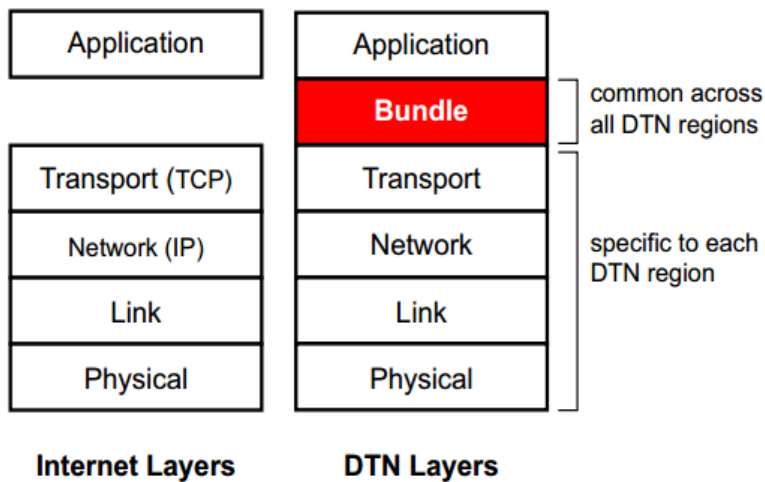

Fig 2: Bundle Layer [4]

Figure 2 shows the bundle layer and its position with respect to the network layers when compared to the internet layers. The DTN bundled layer is common across all the DTN regions while the lower layers are all region specific.

\subsubsection{Store-carry-forward technique}

The concept of store-carry-forward overcomes the problem associated with traditional protocols that may be bidirectional data rates, lack of connectivity, irregular delays etc. This method is very similar as like the real life postal service. Before reaching at the destination a letter has to processed and forwarded through a set of post officers. Like in this technique a complete message or some portion of the message is transferred and successively stored in the nodes until it reaches at the destination through in a network.

As shown in Figure 3 each node has a persistent storage to backup the messages just in case the network fails during transmission.

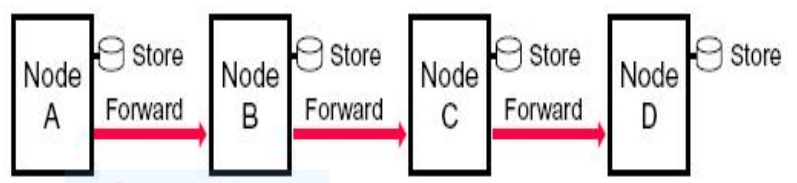

Fig 3: Store and Forward Message Switching [2]

\subsubsection{Types of contacts in DTN}

In DTN, whether or how the nodes make contacts with each other broadly classified as opportunistic and scheduled. The opportunistic contacts are an unscheduled contact which can occur instantly. The node do not have any idea regarding a contact may be direct or indirect in the future. Moving people, automobiles, airplanes etc. make this type of contact and transfer of message as they have sufficient energy for communication. On the other hand in scheduled contact the information is directly or indirectly known to the node for establishment a contact path at a particular time, for a particular duration. Synchronization of time in every node is the major drawback. Example of schedule contact is a interplanetary communication [4].

\subsubsection{Custody transfer}

Bundle layer offered retransmission of message in case-of corrupted or lost data through the concept of bundle layer. The custodian node stores the message until successfully transferred to the next node and takes the custody of next node and takes the custody of that message until TTL expires. 


\section{RELATED WORK}

Here is some recent research work that has been done during past few years.

In 2012 B. F. Ferreira e.t. al. [5] proposed a network management solution for Vehicular delay-tolerant networks (VDTN) using the standard SNMP protocol. A monitoring system is very useful to verify the proper network working, checking possible network anomalies, and to collect statistic data for the network administrator. The SNMP application is embedded with VDTN terminal node application.

In 2013 Zhenjing Zhang, Maode Ma, and Zhigang Jin [6] presented mobility-aware geocast routing algorithm (GeoMob) in which mobile nodes are divided into different clusters according the contact probability of each mobile node. The Spray and Wait algorithm is used for intra-cluster routing, while the social characteristics, centrality of nodes and the network coding technique are used for the intercluster routing.

In 2012 Sherif Tolba, Maram Hakami, Ahmed Mihdhir, Yibo Zhu, Son Le and Jun Hong Cui [7] designed a DTN UWSN (Underwater Sensor Networks) routing protocol and tested properly using real acoustic modems. During the design process, attention was given to the properties related to UWSNs such as the dynamic nature of such networks and the intermittently connected topology. Different combinations of packet types, packet directions, node types, and possible communication scenarios were enumerated and used to assist the protocol design process. The completed version of the protocol was then tested using a virtual modem as well as on real Benthos modems. Results indicate that the protocol is working as expected and the packets succeed to reach the destination.

In 2012 Yue Cao, Zhili Sun and Ning Wang [8] proposed a heuristic routing algorithm for DTNs which sprays the message replication probability rather than copy tickets. The main motivation of this algorithm is to loose the scalability problem of the spray based algorithms, which is to determine the initial value for copy tickets. Based on our defined utility metric for replication probability distribution, the heuristic algorithm dynamically switches between the designed geographic replication phase and replication probability spray phase, according to an encounter angle between pairwise nodes.

In 2012 Nehal Somani, Abhishek Chanda, Samuel C. Nelson and Dipankar Raychaudhuri [9] proposed and evaluated a generalized storage aware local routing protocol for the future Internet. In this they shown how GSTAR can achieve significant performance by combining local link state and DTN style routing. Although omitted from this paper due to space constraints, Author currently has a proof-of-concept implementation of GSTAR running on both the GENI and ORBIT testbeds. In the future they plan to perform a comprehensive evaluation of GSTAR implementation and to make GSTAR more robust to dynamic network conditions by varying its parameters automatically. For example, the threshold for the decision to store or forward should be dynamic.

In 2012 Minsu Huang, Siyuan Chen, Fan Li and Yu Wang [10] Studied reliable topology design problem in a predictable time-evolving DTN with unreliable links modeled by a probabilistic space-time graph. And propose a set of new heuristics which can not only maintain the connectivity and reliability of paths over time but also significantly reduce the cost of topology. Simulation results from random networks demonstrate the efficiency of our methods. This paper presents the first step in exploiting topology design for time evolving DTNs with unreliable links.

In 2012 Sathya Narayanan, Eric McDonald and Geoffrey G. Xie [11] proposed a method to organize various known routing protocols in a spectrum ranging from aggressive to predictive based on a newly defined predictive ability metric. A collection of variations for the two protocols at the two ends of this spectrum, Epidemic and PROPHET.A new mobility model based on random waypoint mobility that increases the propensity for groups of nodes to meet each other more often.

In 2013 Razvan Beuran, Shinsuke Miwa and Yoichi Shinoda [12] presented an emulation testbed intended for DTN application and protocol experiments. Discussed in detail the main changes that were necessary in order to make possible DTN experiments on the QOMB, a generic-purpose wireless network emulation testbed are multi-interface support, fault injection mechanisms, etc.

In 2013 Yue Cao, Zhili Sun, Ning Wang and Fang Yao, Haitham Cruickshank [13] overcome the problem with long delay for information request/ reply due to sparse network density, $\mathrm{CaD}$ (converge-and-diverge) separately replicates message converging and diverging to the movement range estimated for mobile destination by using its historical geographic information. More importantly, $\mathrm{CaD}$ utilizes DR to overcome the limited routing decision and the local maximum problem. The replicated messages are further under prioritized transmission, enhancing routing performance. Although $\mathrm{CaD}$ assumes the constant moving speed in the ideal case, evaluated results in the terms of delivery ratio, overhead ratio, and average delivery latency. Regarding real-world implementation, the factor of GPS error should be taken into account.

In 2013 Qiao Fu, Bhaskar Krishnamachari and Lin Zhang [14] firstly proposed a model that fully manifests the special features of vehicular DTN with consideration of the limited local channel capacity caused by wireless interference. Further propose a heuristic and accurate network performance measurement for the large scale DTN which evaluates the network throughput within deadline. A routing scheme for DTN, called Density Adaptive routing With Node deadline awareness (DAWN) is then introduced to improve the packet delivery ratio within deadline where network capacity is constraint. The core idea of DAWN is to adjust forward strategy and protocol parameters based on local node density with the awareness of local channel capacity. In DAWN, a utility value suggesting the delivery probability of the packets within time constraint is maintained for each packet by each mobile node. Mobile nodes in each cell decide how many packets to broadcast and which packet to broadcast independently based on information of local density and the packet utility gain.

In 2013 Tiansi $\mathrm{Hu}$ and Yunsi Fei [15] proposes a novel adaptive and energy- efficient routing protocol for underwater delay/disruption tolerant sensor networks, with predictions based on LMS filters and routing supported by a Q-learning algorithm. The sensor nodes are able to predict the future contact events precisely and estimate how much delay is expected for different neighbors through on-line Q-learning, without any prior assumptions of geographical information and node mobility patterns. 
In 2014 Lei Zhang, Boyang Yu and Jianping Pan [16] proposes an advanced mobility-aware geocast (Ge-oMob) scheme is proposed for large-scale urban VANETs. This new scheme has many new features compared with the existing geocast schemes: first, it is designed from a DTN point of view, it is enable when transient connectivity and high mobility in VANETs. Second, In vehicle mobility different levels and aspects is employed to make the GeoMob simple, scalable, communication and computation-effective; third, practical issues are well considered by introducing the analysis of real trace, that is conducted in a simulation in efficient buffer management.

\subsection{Summary}

It is observed that within the DTN routing, still there is scope of research. In [5] SNMP is specially design for VDTN kind of network environment that does not perform well for conventional network environment. In [6] different techniques may be used for inter and intra cluster to get effective result.

The approach used in [8] uses heuristic based routing algorithm which has problem of local maxima (best path from source to destination or good candidate to forward a messages) but there may be other shortest path available to forward a messages. It can be made efficient by using optimal path selection. In [9] Mobility related challenges such as link quality variation, node disconnection, and node partitioning are solved on NS3 based Simulator. So can be overcomed on NS2 and ONE also.

\section{CONCLUSION}

During the literature survey, it is observed that the problem in DTN is Lack of continuous end-to-end connection due to node mobility, All nodes has constrained power source, and limited space to storage of data. There is also problem in a data packet delivery and source to reach destination overhead in the network. In network, based on grouping there is uses inclusive social metrics which in result, collect wide information about network to better predict the path to destination.

To overcome the frequent disconnection, DTN nodes are required to store data packets for long period of time until they contact with other nodes. To increase delivery probability spread multiple copies of packets in network so that one of them reaches at destination

Social grouping maximizes data delivery while minimizes network overhead by efficiently spreading number of message copies in the network.

\section{REFERENCES}

[1] S. Patil and G. R. Chillerge, "Delay Tolerant NetworksSurvey Paper," Int. Journal of Engineering Research and Applications, vol. 4, no. 2, pp. 21-25, February 2014.

[2] W. Kehr, "Delay Tolerant Network," University of Missouri-Rolla, May 2006. [Online]. Available: http://web.mst.edu/ mobildat/DTN/index.html. [Accessed November 2016].

[3] V. Cerf, S. Burleigh, A. Hooke, L. Torgerson, R. Durst, K. Scott, K. Fall and H. Weiss, "Delay-Tolerant Networking Architecture," Network Working Group, April 2007. [Online]. Available: https://tools.ietf.org/html/rfc4838.html. [Accessed November 2016].
[4] P. K. Gantayat and S. Jena, "Delay Tolerant Network - A Survey," International Journal of Advanced Research in Computer and Communication Engineering, vol. 4, no. 7, July 2015 .

[5] B. F. Ferreira, J. N. Isento, J. A. Dias, J. P. Rodrigues and L. Zhou, "An SNMP based solution for vehicular delay-tolerant network management," in Global Communications Conference (GLOBECOM), 2012.

[6] Z. J. Zhenjing Zhang and M. Ma, "CCS-DTN:Efficient Routing in Social DTNs Based on Clustering and Network Coding," in Global Communications Conference (GLOBECOM), 2013.

[7] S. Tolba, M. Hakami, A. Mihdhir, Y. Zhu, S. Lee and J. H. Cui, "Underwater Delay tolerant Routing in Action," Oceans, pp. 1-6, 2012.

[8] Y. Cao, Z. Sun and N. Wang, "Spraying the Raplication Probability with geographic Assistance for Delay Tolerant Networks," in Internation Conference on Communications (ICC), 2012.

[9] N. Somani, A. Chanda, S. C. Nelson and D. RayChaudhuri, "Storage aware routing protocol for robust and efficient services in the future mobile Intrenet," in International Conference on Communications (ICC), 2012.

[10] M. Huang, S. Chen, F. Li and Y. Wang, "Topology design in time-evolving delay-tolerant networks with unreliable links," in Global Communications Conference (GLOBECOM), 2012.

[11] S. Narayanan, E. McDonald and G. G. Xie, "A Framework for Simulation Analysis of Delay Tolerant Routing Protocols," in Vehicular Technology Conference (VTC Fall), 2012.

[12] R. Beuran, S. Miwa and Y. Shinoda, "Network emulation testbed for DTN applications and protocols," in INFOCOM, 2013.

[13] Y. Cao, Z. Sun, N. Wang, F. Yao and H. Cruickshank, "Converge-and-Diverge: A Geographic Routing for Delay/Disruption-Tolerant Networks Using a Delegation Replication Approach," in IEEE Transactions on Vehicular Technology, 2013.

[14] Q. Fu, B. Krishnamachari and L. Zhang, "DAWN: A density adaptive routing for deadline-based data collection in vehicular delay tolerant networks," Tsinghua Science and Technology, vol. 18, no. 3, pp. 230-241, 2013.

[15] T. Hu and Y. Fei, "An adaptive routing protocol based on connectivity prediction for underwater disruption tolerant networks," in Global Communications Conference (GLOBECOM), 2013.

[16] L. Zhang, B. Yu and J. Pan, "GeoMob: A mobility-aware geocast scheme in metropolitans via taxicabs and buses," in INFOCOM, 2014.

[17] P. Puri and M. P. Singh, "A Survey Paper on Routing in Delay-tolerant Network," in International conference on Information Systems and Computer Networks, 2013. 